\title{
Ansatzpunkte für die Prävention frühen jugendlichen Alkoholkonsums
}

\author{
Frühkindliche Risikofaktoren, Trinkmotive und \\ Mutter-Jugendlichen-Übereinstimmung
}

\author{
Max Supke und Wolfgang Schulz
}

Abteilung für Klinische Psychologie, Psychotherapie und Diagnostik, Institut für Psychologie, Technische Universität Braunschweig

\begin{abstract}
Zusammenfassung: Ziele: In der vorliegenden Studie werden sowohl frühkindliche als auch elterliche Risikofaktoren, die jugendliche Trinkmotivation und die Übereinstimmung zwischen den Müttern und Jugendlichen hinsichtlich des jugendlichen Alkoholkonsums zur Ableitung von präventiven Ideen ausgewertet. Methodik: Die Daten von 239 Familien aus dem längsschnittlichen deutschen Projekt Zukunft Familie wurden vom Kindergartenalter ( $M=4.5 \mathrm{Jahre}$ ) bis in das Jugendalter $(M=14 \mathrm{Jahre}$ ) erhoben. Mittels binär logistischer Regressionsmodelle wurden die Zusammenhänge zwischen psychischen Auffälligkeiten im Kindesalter, der soziodemographischen Situation sowie Verhaltensweisen der Eltern und dem Alkoholkonsum der Kinder zehn Jahre später analysiert. Ergebnisse: Kinder von Müttern mit einer höheren Schulbildung und aus Familien mit einem höheren sozialen Status hatten ein erhöhtes Risiko, schon vor dem gesetzlich erlaubten Mindestalter, regelmäßig Alkohol zu trinken. Bei den im Durchschnitt 4.5-jährigen Kindern erwiesen sich internalisierende Auffälligkeiten als Schutzfaktor vor einem frühzeitigen jugendlichen Konsum. Alkohol wurde im Jugendalter vor allem aus sozialen und Verstärkungsmotiven getrunken. Mütter unterschätzten signifikant den Konsum ihrer Kinder um das Vier- bis Fünffache. Schlussfolgerung: Präventionsmaßnahmen könnten den Jugendlichen Alternativen aufzeigen, um die sozialen und verstärkenden Motivationen auf risikofreien Wegen zu erreichen. Zur verbesserten Einschätzung des kindlichen Konsums, könnten Mütter in Hinweisen geschult werden, die auf einen übermäßigen Konsum hindeuten. Das erhöhte Risiko von Kindern aus Familien mit einem höheren sozialen Status könnte dabei berücksichtigt werden.
\end{abstract}

Schlüsselwörter: Prävention, Trinkmotive, kindliche Risikofaktoren, Längsschnitt, Übereinstimmung

Approaches to Preventing Early Adolescent Alcohol Use: Early Childhood Risk Factors, Drinking Motives, and Mother-Adolescent Agreement

Abstract: Purpose: In the present study, both early childhood- as well as parental risk factors, adolescent drinking motives, and maternal-adolescent agreement on adolescent alcohol use are analyzed to generate approaches to prevention. Method: Data from 239 families from the longitudinal German project Future Family were collected from kindergarten age ( $M=4.5$ years) to adolescence ( $M=14$ years). Binary logistic regression models were used to calculate the relationships between mental health problems in childhood, sociodemographic conditions, parental behaviours, and children's consumption of alcohol ten years later. Results: Children of mothers with a higher level of school education and those coming from families with a higher social status had an increased risk of drinking alcohol regularly prior to reaching the legally permitted minimum age. Alcohol consumption during adolescence was primarily due to social- and reinforcement motives, whilst internalizing mental health problems proved to be a protective factor. Mothers significantly underestimated their children's alcohol consumption by a factor of four to five. Conclusion: Preventive interventions could show adolescents how they could reach social- and reinforcing motives in ways that are less risky than by drinking alcohol. To improve mothers' estimation of children's consumption behaviours, training could be offered to improve the recognition of cues indicating problematic alcohol use. The increased risk of children from families with a higher social status could be taken into account.

Keywords: prevention, drinking motives, childhood risk factors, longitudinal, agreement

Der Alkoholkonsum Jugendlicher in Deutschland ist weit verbreitet. So ergab z.B. die Drogenaffinitätsstudie der Bundeszentrale für gesundheitliche Aufklärung (BZgA) aus dem Jahr 2019, dass $63 \%$ der 12- bis 17-Jährigen bereits Alkohol getrunken haben und $9 \%$ regelmäßig Alko- hol konsumieren. Von Rauscherfahrungen innerhalb der letzten 30 Tage berichten $15 \%$ der Jugendlichen, während rund $4 \%$ bereits (für Erwachsene) gesundheitlich riskante Alkoholmengen konsumieren (Orth \& Merkel, 2020). 
Der Konsum von Alkohol im Jugendalter steht dabei in Zusammenhang mit gesundheitlichen Risiken sowie Beeinträchtigungen der gesunden Entwicklung des Gehirns (Meruelo, Castro, Cota \& Tapert, 2017). Jungen scheinen dabei in Deutschland bereits früher regelmäßiger Alkohol zu konsumieren als Mädchen (Lampert \& Thamm, 2007). Weiterhin ist der Konsum von Alkohol im Jugendalter mit psychischen Beschwerden assoziiert (Brownlie et al., 2019). Jugendliche, die Alkohol vor dem Alter von 15 Jahren konsumieren, haben in ihrem späteren Leben ein signifikant erhöhtes Risiko eine Alkoholabhängigkeit $(O R=$ 1.38) zu entwickeln oder Alkohol zu missbrauchen $(O R=$ 1.52; Dawson, Goldstein, Chou, Ruan \& Grant, 2008). Prävention sollte aus diesen Gründen bereits möglichst frühzeitig ansetzen.

Soziodemographische Merkmale und Faktoren aus der frühen Kindheit scheinen eine bedeutsame Rolle bezüglich des jugendlichen Konsumverhaltens zu spielen. Familiäre (z.B. negative Eltern-Kind-Beziehung), elterliche (z.B. Substanzmissbrauch, schlechte Beziehungsqualität) aber auch kindliche Faktoren (z. B. Verhaltensauffälligkeiten) erwiesen sich schon in der frühen Kindheit als potentielle Risikofaktoren für den späteren Alkohol- und Drogenkonsum (Maggs, Patrick \& Feinstein, 2008; Reinherz, Giaconia, Hauf, Wasserman \& Paradis, 2000).

Um adäquate Präventionsprogramme zu entwickeln, sollten die Motive des Alkoholkonsums ebenfalls berücksichtigt werden. Es zeigte sich für deutsche Jugendliche, dass soziale Motive („Zusammenkünfte mehr genießen“) am stärksten bei ihnen ausgeprägt sind, gefolgt von Verstärkungs- („Spaß haben“), Bewältigungs- („Probleme vergessen“) und Konformitätsmotiven (,dazugehören“) (Kuntsche \& Kuntsche, 2009).

Ein weiterer potenzieller Ansatzpunkt für Präventionsmaßnamen ist, dass Eltern den Konsum ihrer Kinder in Deutschland unterschätzen. Besonders problematisch ist dabei, dass die Abweichungen umso größer sind, je jünger das Kind ist, je häufiger es Alkohol konsumiert und je hochprozentiger die Getränke sind (Lampert \& Thamm, 2007). Somit stellt die Verbesserung der elterlichen Einschätzung einen weiteren geeigneten Ansatzpunkt dar.

Die Studienlage zu frühkindlichen Risikofaktoren, den Trinkmotiven sowie zur Übereinstimmung der elterlichen und jugendlichen Einschätzung des jugendlichen Alkoholkonsums in Deutschland ist gering. Anhand der Daten aus einer 10-Jahres-Längsschnittstudie soll versucht werden, die Studienlage zu verbessern und neue Erkenntnisse in diesen Bereichen zu generieren, um im Anschluss präventive Maßnahmen abzuleiten.

\section{Fragestellungen}

Wenn im Folgenden von Kindern gesprochen wird, sind damit Kinder im Kindergartenalter zwischen drei und sechs Jahren gemeint, während sich die Bezeichnung des Jugendlichen auf das Alter zwischen 12 und 17 Jahren bezieht. Die Studie fokussiert sich auf die folgenden Fragestellungen:

Fragestellung 1: Unterscheidet sich der frühe jugendliche Alkoholkonsum in Abhängigkeit vom Geschlecht, der sozialen Schicht, dem Erziehungsstatus (Ein- vs. Zwei-Eltern-Familien) und dem Migrationshintergrund?

Fragestellung 2: Lässt sich aufgrund des Vorhandenseins von kindlichen (internalisierende/externalisierende Auffälligkeiten, Temperament) und elterlichen Risikofaktoren (dysfunktionales Erziehungsverhalten, psychische Belastung der Mutter, Vorhandensein von Alkoholproblemen von Mutter und/oder Vater) im frühen Kindesalter der Konsum von Alkohol im Jugendalter vorhersagen?

Fragestellung 3: Welche Trinkmotive sind beim jugendlichen Alkoholkonsum am weitesten verbreitet?

Fragestellung 4: Stimmen die Einschätzungen der Jugendlichen und ihrer Mütter hinsichtlich des jugendlichen Alkoholkonsums überein?

\section{Methode}

\section{Studiendesign und Durchführung}

Die Erhebungen erfolgten im Rahmen der DFG-Projekte Zukunft Familie I (ZF I) und Zukunft Familie III (ZF III; s. Hahlweg \& Schulz, 2018). Bei der Studie ZF I handelt es sich um eine randomisierte Kontrollgruppenstudie, in der die Wirksamkeit des Elterntrainings Positive Parenting Program (Triple P; Sanders, 2012) untersucht wurde. Der Studie ZF III liegt ein Längsschnittdesign zugrunde, bei dem die Vorhersage von Verhaltensauffälligkeiten im Jugendalter (10-Jahres Follow-up [FU10]) unter Berücksichtigung von Risiko- und Schutzfaktoren im Kindergartenalter (Prä, ZF I) im Vordergrund stand.

Während eines Hausbesuches wurden der Jugendliche und ein Elternteil in parallel stattfindenden Gesprächen getrennt voneinander befragt. Die Familie konnte entscheiden, ob das Interview mit der Mutter oder dem Vater durchgeführt wurde. Der Großteil der Familien (94\%), welche in dieser Studie analysiert werden, entschied sich dazu, dass die Mutter interviewt wurde. Zur Datenerhebung wurde eine Kombination aus Interview und elektronischer standardisierter Befragung gewählt. Der Hausbesuch dauerte pro Familie maximal 2.5 Stunden. Bei den Interviewenden handelte es sich in der Regel um Diplombzw. Master-Psycholog_innen, welche sich mindestens in 
der Ausbildung zu Psychologischen Psychotherapeut_innen befanden. Sie wurden vorab durch ein intensives Training auf die Gespräche mit den Familien vorbereitet. Die Familien erhielten eine Vergütung von insgesamt $80 €$ (je $40 €$ für den Jugendlichen und das jeweilige Elternteil).

\section{Stichprobenrekrutierung und -beschreibung}

Mithilfe des Kita-Sozialstrukturindex (= Maß der soziodemografischen Struktur des Einzugsbereiches) und der Anzahl der Kinder wurden 17 zufällig ausgewählte Kitas in Braunschweig parallelisiert und anschließend randomisiert. Innerhalb der Kitas konnten 280 Familien rekrutiert werden. Die Bedingung für die Teilnahme war ein ausreichendes Grundverständnis der deutschen Sprache.

Für die Follow-Up-Erhebung 10 Jahre später (FU10) konnten noch 249 Familien gewonnen werden (Ausschöpfungsrate: $89 \%$ ). Zu Prä fehlten von drei Familien Fragebögen. Weiterhin fehlten zu FU10 von sieben Familien Einschätzungen des jugendlichen Alkoholkonsums. Diese $n=10$ Familien werden von den folgenden Analysen ausgeschlossen, sodass sich ein finales $N=239$ ergibt.

Zum Zeitpunkt der Follow-up-Erhebung (von 20112013) waren die Jugendlichen durchschnittlich 14 Jahre $(S D=1.2)$, die Mütter 45 Jahre $(S D=4.8)$ und die Väter 49 Jahre $(S D=5.4)$ alt. Rund $54 \%$ der Jugendlichen waren Jungen und $56 \%$ der Jugendlichen besuchten ein Gymnasium. Von den Müttern besaßen 1\% keinen Abschluss, $9 \%$ den Hauptschulabschluss, 33\% die mittlere Reife und $57 \%$ das Abitur, bei den Vätern hatten $1 \%$ keinen Abschluss, $12 \%$ den Hauptschulabschluss, $22 \%$ die mittlere Reife und $65 \%$ das Abitur. Jede zehnte Mutter (11\%) war alleinerziehend und $8 \%$ der Familien wiesen einen Migrationshintergrund auf. Es gab keine alleinerziehenden Väter in dieser Stichprobe. Innerhalb der Stichprobe hatten $54 \%$ der Familien am Triple P teilgenommen.

\section{Messinstrumente}

Innerhalb der Interviews wurden Daten zur Soziodemographie (bspw. Alter, Migrationshintergrund, Erziehungsstatus) und zum Alkoholkonsum erhoben.

\section{Alkoholkonsum (FU10)}

Anhand der Selbsteinschätzung auf die Fragen: „Hast Du selbst schon einmal Alkohol getrunken?" und „Trinkst Du regelmäßig Alkohol, das heißt, mindestens einmal pro Woche?" wurden die Jugendlichen in die drei Gruppen: Noch nie, nur mal probiert/ab und zu (weniger als einmal pro Wo- che) und regelmäßiger Konsum (mindestens einmal pro Woche) eingeteilt. Sowohl die Jugendlichen selbst als auch das befragte Elternteil wurden nach der Anzahl der Trinktage des Kindes (Anzahl an Tagen pro Monat) und anschließend nach der Trinkmenge (z.B. kleine, große Gläser) sowie der Sorte (bspw. Bier oder Wein) gefragt. Die Fragen orientierten sich insgesamt an denen der regelmäßig stattfindenden repräsentativen Befragungen der Bundeszentrale für gesundheitliche Aufklärung (Orth \& Merkel, 2020).

Die befragten Elternteile gaben zusätzlich zu Aspekten des vermuteten Konsums ihres Kindes und den Regeln im Umgang mit Alkohol Auskunft (bspw. „Gibt es für Ihr Kind Regeln, was den Umgang mit Alkohol angeht?").

\section{Mütterliche psychische Belastung}

Die Depression Anxiety Stress Scales (DASS) sind ein Selbstauskunftsinstrument, welche mittels 42 Items und den drei Skalen depressive Gestimmtheit, Angst und Stressbelastung sowie einem Gesamtwert die psychische Belastung der Mütter erfassen (Köppe, 2001). Höhere Werte sind ein Indiz für vermehrte psychische Beschwerden der Mütter am Prä-Messzeitpunkt $(\alpha=.94)$.

\section{Mütterliches Erziehungsverhalten}

Die Anwendung von dysfunktionalen Erziehungspraktiken durch die Mütter wurde mit den 35 Items des Erziehungsfragebogens (EFB) erfasst (Naumann et al., 2010). Höhere Gesamtwerte innerhalb des Selbstauskunftsfragebogens repräsentieren ein dysfunktionaleres mütterliches Erziehungsverhalten zu Prä $(\alpha=.87)$.

\section{Elternfragebogen über das Verhalten von Klein- und Vorschulkindern (CBCL 1 1 1/2-5) bzw. von Kindern und Jugendlichen (CBCL 4-18)}

Die CBCL $1 \frac{1 / 2-5}{2}$ und $4-18$ beschreibt in 100 (bzw. 118) Items Aussagen über das Vorhandensein verschiedener kindlicher Verhaltensprobleme, welche von den Müttern auf einer dreistufigen Skala ( 0 = „nicht zutreffend“, 1 = „manchmal zutreffend“, 2 = „häufig zutreffend“) eingeschätzt werden (Döpfner, Plück, Kinnen \& Arbeitsgruppe Deutsche Child Behavior Checklist, 2014). Der Fragebogen $(\alpha=.84-.95)$ besteht aus einem Gesamtwert sowie den Skalen Internalisierende (z.B. depressive Symptome) und Externalisierende Auffälligkeiten (z.B. ADHS-Symptome). Höhere Werte auf den Skalen sind ein Indiz für vermehrte kindliche psychische Auffälligkeiten im jeweiligen Bereich am Prä-Messzeitpunkt.

\section{Kindliches Temperament}

Das kindliche Temperament im Kleinkindalter wurde durch die Mütter anhand von neun Items, welche zu zwei Skalenwerten zusammengefasst wurden, erfasst. Die erste Skala „Annäherung/Exploration“ ( $\alpha=.64)$ bestand dabei 
aus vier Items (bspw. „Das Kind gewöhnt sich schnell an neue Menschen.“), während die zweite Skala „Unruhe/Ärger“ ( $\alpha=.72)$ fünf Items (bspw. „Das Kind war ständig in Bewegung.") beinhaltete. Höhere Summenwerte zu Prä beschreiben ein ausgeprägteres Temperament des Kindes im jeweiligen Bereich (Lichtsinn, 2013).

\section{Jugendliche Trinkmotive}

Das vorherrschende Trinkmotiv der Jugendlichen wurde durch Selbstangaben auf den 12 Items der Kurzform des Drinking-Motive-Questionnaire-Revised (DMQ-R SF; Kuntsche \& Kuntsche, 2009) ermittelt. Der Fragebogen umfasst jeweils drei Items der vier Trinkmotivations-Skalen: soziale, Verstärker-, Bewältigungs- und Konformitätsmotive. Höhere Werte auf den Skalen sprechen für eine ausgeprägtere Trinkmotivation.

\section{Statistische Auswertung}

Zunächst wurden die Daten deskriptiv ausgewertet. Zur Beantwortung der Fragestellungen 1 und 2, welche die Vorhersage des Alkoholkonsums im Jungendalter anhand von soziodemographischen und frühkindlichen Faktoren fokussieren, wurden binär logistische Regressionsmodelle erstellt. Zur Auswertung der Trinkmotive (Fragestellung 3) wurden Mittelwerte und Partialkorrelationen berechnet. Der Vergleich der mütterlichen und jugendlichen Einschätzungen (Fragestellung 4) der Konsummenge erfolgte durch Mittelwertsvergleiche (Wilcoxon-Test) und die Berechnung von Zusammenhängen (Spearman-Korrelationen). Zur Bestimmung der Konsummenge wurde der Frequenz-Menge-Index (FMI) berechnet. Für jede Getränkesorte wurde das Produkt aus der Menge pro Konsumtag und Anzahl der Konsumtage gebildet. Das Produkt wurde in die Menge an reinem Alkohol (in Gramm) umgerechnet und für alle Getränkesorten aufsummiert. Anschließend wurde die Konsummenge von reinem Alkohol pro Tag in einem Monat errechnet (Orth \& Merkel, 2020). Das Alter der Jugendlichen wurde dabei in den Analysen, soweit es nötig war, statistisch kontrolliert. Für die Signifikanztestung wurde ein Alphaniveau von $p=$ .05 gemäß der üblichen Konvention angenommen.

\section{Ergebnisse}

\section{Deskription}

Von den Jugendlichen in dieser Studie hatten 38\% $(n=90$; Jungen: 39\%, Mädchen: 36\%) noch nie Alkohol konsumiert, $16 \%$ ( $n=38$; Jungen: $13 \%$, Mädchen: $19 \%)$ schon mal Alkohol probiert bzw. tranken hin und wieder und $46 \%$ ( $n=111$; Jungen: $48 \%$, Mädchen: $45 \%$ ) tranken regelmäßig (Tabelle 1). Rund $71 \%$ der befragten Eltern gaben an, dass es Regeln für ihr Kind im Umgang mit Alkohol gab.

Das Erstkonsumalter der regelmäßig Trinkenden innerhalb dieser Stichprobe $(n=111)$ betrug 13.5 Jahre $(S D=$ 1.5). Bier $(M=1.6 ; S D=2.0)$ und Mixgetränke $(M=1.6$; $S D=2.5)$ wurden dabei im Mittel an einem Tag mehr im Monat getrunken als Wein/Sekt $(M=0.6 ; S D=1.0)$, Schnaps $(M=0.5 ; S D=1.0)$ und Cocktails $(M=0.5 ; S D=$ 0.9). Im Durchschnitt wurden täglich $3.3 \mathrm{~g}(S D=3.7)$ reiner Alkohol von den regelmäßig Trinkenden konsumiert. Weiterhin berichteten $31 \%$ von Rauscherfahrungen in den letzten drei Monaten und 25\% hatten Binge Drinking in den letzten 30 Tagen betrieben. Die häufigsten Folgen des Konsums, welche von den regelmäßig Konsumierenden berichtet wurden, waren Erinnerungslücken (38\%), Reuegefühle (32\%) und Konfrontationen mit dem Trinkverhalten $(24 \%)$.

\section{Soziodemographische Unterschiede (Fragestellung 1)}

Mit einem binär logistischen Regressionsmodell wurde überprüft, ob sich ein regelmäßiger (vs. noch nie getrunken/nicht regelmäßig) jugendlicher Alkoholkonsum durch soziodemographische Merkmale (Prä) längsschnittlich vorhersagen lässt. Das Modell geht mit einer guten Modellanpassung einher (Tabelle 2). Signifikante Zusammenhänge mit kleinen und mittleren Effektstärken ergaben sich zum Alter $(B=1.25 ; p<.001 ; O R=3.48 ; d=0.69)$ und zur sozialen Schicht (Kita-Sozialstrukturindex: $B=$ $0.77 ; p=.019 ; O R=2.16 ; d=0.43$; Schulabschluss Mutter: $B=0.83 ; p=.011 ; O R=2.30 ; d=0.46)$, derart, dass Jugendliche aus höheren Schichten ein erhöhtes Risiko für einen regelmäßigen Alkoholkonsum hatten. Die Zusammenhänge zu den Merkmalen Erziehungsstatus, Geschlecht und Migrationshintergrund wurden in dieser Stichprobe hingegen nicht signifikant.

\section{Risiko- und Schutzfaktoren im Kindesalter (Fragestellung 2)}

In Hinblick auf Fragestellung 2 wurde untersucht, inwiefern Risikofaktoren im Kindesalter (Prä) mit dem regelmäßigen jugendlichen Alkoholkonsum (vs. noch nie getrunken/nicht regelmäßig) nach 10 Jahren zusammenhängen. Das Alter sowie das Geschlecht der Jugendlichen und die Teilnahme der Eltern am Triple P wurden in dem binär logistischen Regressionsmodell statistisch kontrolliert. 
Tabelle 1. Deskription des Alkoholkonsums der Jugendlichen

\begin{tabular}{|c|c|c|c|c|}
\hline & & Bezugsstichprobe & N & $\%$ \\
\hline Alkoholkonsum & $\begin{array}{l}\text { - noch nie getrunken } \\
\text { - probiert, hin und wieder } \\
\text { - regelmäßiger Konsum }\end{array}$ & $N=239$ & $\begin{array}{r}90 \\
38 \\
111\end{array}$ & $\begin{array}{l}38 \\
16 \\
46\end{array}$ \\
\hline Elterliche Regeln für das Konsumverhalten & $\begin{array}{l}\text { - es gibt keine Regeln } \\
\text { - es gibt Regeln }\end{array}$ & $N=239$ & $\begin{array}{c}70 \\
169\end{array}$ & $\begin{array}{l}29 \\
71\end{array}$ \\
\hline Erstkonsumalter & & $\begin{array}{l}N=111 \\
\text { (regelmäßiger Konsum) }\end{array}$ & \multicolumn{2}{|c|}{$M=13.5, S D=1.5$} \\
\hline Trinktage (in einem Monat) & $\begin{array}{l}\text { - Bier } \\
\text { - Wein/Sekt } \\
\text { - Schnaps } \\
\text { - Cocktails } \\
\text { - Mixgetränke }\end{array}$ & $\begin{array}{l}N=111 \\
\text { (regelmäßiger Konsum) }\end{array}$ & \multicolumn{2}{|c|}{$\begin{array}{l}M=1.6, S D=2.0 \\
M=0.6, S D=1.0 \\
M=0.5, S D=1.0 \\
M=0.5, S D=0.9 \\
M=1.6, S D=2.5\end{array}$} \\
\hline \multirow[t]{2}{*}{$\begin{array}{l}\text { Frequenz-Menge-Index (FMI; Reinalkohol in Gramm/ } \\
\text { Tag) }\end{array}$} & Elterneinschätzung & $\begin{array}{l}N=111 \\
\text { (regelmäßiger Konsum) }\end{array}$ & \multicolumn{2}{|c|}{$M=0.6, S D=1.4$} \\
\hline & Jugendlicheneinschätzung & $\begin{array}{l}N=111 \\
\text { (regelmäßiger Konsum) }\end{array}$ & \multicolumn{2}{|c|}{$M=3.3, S D=3.7$} \\
\hline Rauscherfahrung & $\begin{array}{l}\text { - Lebenszeitprävalenz } \\
\text { - innerhalb der letzten } 3 \text { Monate }\end{array}$ & $\begin{array}{l}N=111 \\
\text { (regelmäßiger Konsum) }\end{array}$ & $\begin{array}{l}50 \\
34\end{array}$ & $\begin{array}{l}45 \\
31\end{array}$ \\
\hline Binge Drinking & $\begin{array}{l}\text { - in den letzten } 30 \text { Tagen } \\
\text { - > 4-mal im Monat }\end{array}$ & $\begin{array}{l}N=111 \\
\text { (regelmäßiger Konsum) }\end{array}$ & $\begin{array}{r}28 \\
4\end{array}$ & $\begin{array}{r}25 \\
4\end{array}$ \\
\hline \multirow[t]{3}{*}{ Folgen (mindestens ein Mal) } & $\begin{array}{l}\text { - Erinnerungslücken } \\
\text { - Konfrontation mit dem Trink- } \\
\text { verhalten }\end{array}$ & \multirow[t]{3}{*}{$\begin{array}{l}N=111 \\
\text { (regelmäßiger Konsum) }\end{array}$} & $\begin{array}{l}42 \\
27\end{array}$ & $\begin{array}{l}38 \\
24\end{array}$ \\
\hline & $\begin{array}{l}\text { - Körperliche Auseinanderset- } \\
\text { zungen }\end{array}$ & & 8 & 7 \\
\hline & - Verhalten bereuen & & 36 & 32 \\
\hline
\end{tabular}

Tabelle 2. Analysen der Soziodemographie (Prä): Ergebnisse der multivariaten binären logistischen Regression (noch nie getrunken/nicht regelmäBiger vs. regelmäßiger) Alkoholkonsum

\begin{tabular}{|c|c|c|c|c|c|c|c|c|}
\hline & $B$ & $S D$ & Wald & $p$ & $O R$ & $d$ & $95 \%-\mathrm{KI}$ OR & VIF \\
\hline Alter & 1.25 & 0.2 & 47.2 & $.000 * \star \star$ & 3.48 & 0.69 & $2.4-5.0$ & 1.04 \\
\hline $\begin{array}{l}\text { Geschlecht } \\
(0=\text { Mädchen, } 1 \text { = Junge })\end{array}$ & 0.30 & 0.3 & 0.9 & .339 & 1.35 & 0.17 & $0.7-2.5$ & 1.02 \\
\hline $\begin{array}{l}\text { Kita-Sozialstrukturindex } \\
(0=\text { untere, } 1=\text { höhere Schicht) }\end{array}$ & 0.77 & 0.3 & 5.5 & $.019 *$ & 2.16 & 0.43 & $1.1-4.1$ & 1.05 \\
\hline $\begin{array}{l}\text { Schulbildung Mutter } \\
(0=\text { niedrige, } 1 \text { = höhere) }\end{array}$ & 0.83 & 0.3 & 6.4 & $.011 \star$ & 2.30 & 0.46 & $1.2-4.4$ & 1.05 \\
\hline $\begin{array}{l}\text { Erziehungsstatus } \\
(0=\text { Zwei-Eltern-Familie, } 1 \text { = Ein-Eltern-Familie })\end{array}$ & 0.64 & 0.4 & 2.2 & .142 & 1.90 & 0.35 & $0.8-4.5$ & 1.06 \\
\hline $\begin{array}{l}\text { Migrationshintergrund } \\
(0=\text { ohne, } 1=\text { mit) }\end{array}$ & 0.14 & 0.6 & 0.1 & .804 & 1.16 & 0.08 & $0.4-3.6$ & 1.03 \\
\hline Omnibus $\chi^{2}=77.4, p<.001$, Nagelkerkes $\mathrm{R}^{2}=.370$ & HL-Tes & $=8.2$ & $413, k$ & te Vorhe & se: 71. & & & \\
\hline
\end{tabular}

Anmerkungen. ${ }^{*} p<.05,{ }^{* *} p<.01,{ }^{* *} p<.001 ; \mathrm{VIF}$ : Varianz-Inflations-Faktoren. 
Auch dieses Modell weist eine gute Modellanpassung auf (Tabelle 3). Signifikante Effekte wurden lediglich beim Alter $(B=1.21 ; p<.001$; OR $=3.35 ; d=0.67)$ und bei den internalisierenden Auffälligkeiten im Kindergartenalter $(B=-0.06, p=.014, \mathrm{OR}=0.94, d=-0.03)$ vorgefunden. Internale kindliche Auffälligkeiten scheinen in geringem Maße vor einem regelmäßigen Konsum im Jugendalter zu schützen. Keine signifikanten Vorhersagen zeigten sich in Hinblick auf das biologische Geschlecht, externale Auffälligkeiten, das kindliche Temperament, das mütterliche dysfunktionale Erziehungsverhalten, die psychische mütterliche Belastung und familiäre Alkoholprobleme.

\section{Trinkmotive der regelmäßig Konsumierenden (Fragestellung 3)}

Um das vorherrschende Trinkmotiv für die regelmäßig Konsumierenden $\mathrm{zu}$ bestimmen, wurden die Summenwerte für die vier Skalen des DMQ-R SF ermittelt. Von sechs Jugendlichen lagen keine Fragebögen vor, sodass sich die folgenden Analysen auf $n=105$ beziehen.

Sowohl die sozialen Motive $(M=7.9 ; S D=3.6)$ als auch die Verstärkungsmotive $(M=6.4 ; S D=3.1)$ waren am stärksten ausgeprägt. Bewältigungs- $(M=4.1 ; S D=2.1)$ und Konformitätsmotive $(M=3.7 ; S D=1.5)$ spielten demgegenüber eine geringere Rolle.
Sowohl soziale Motive $(r=.34 ; p<.001)$ als auch Verstärkungs- $(r=.45 ; p<.001)$ und Bewältigungsmotive $(r=$ $.30 ; p=.002)$ korrelierten signifikant positiv mit dem FMI aus der Sicht der Jugendlichen. Konformitätsmotive $(r=$ $.14 ; p=.144$ ) wiesen hingegen keinen signifikanten Zusammenhang auf. Das Alter wurde bei der Berechnung der Partialkorrelationen statistisch kontrolliert.

\section{Übereinstimmung der Mutter- und Jugendlicheneinschätzung (Fragestellung 4)}

Die Angaben der $n=14$ befragten Väter wurden aufgrund der geringen Anzahl von diesen Analysen ausgeschlossen, sodass sich die Berechnung nur auf die Einschätzungen der $n=225$ Mütter beziehen. Die Mittelwertsvergleiche und Korrelationen wurden getrennt für die Gruppe derjenigen, die bisher Alkohol getrunken hatten (Gruppe 1; regelmäßig und schon mal Alkohol probiert; $n=140$ ), und für die Gruppe der regelmäßig Trinkenden (Gruppe 2; $n=103$ ) berechnet. Die Ergebnisse zeigen, dass die Mütter den Konsum von reinem Alkohol (in Gramm) ihrer Kinder sowohl in Gruppe 1 (Mütter: $M=0.6, S D=1.3$; Jugendliche: $M=2.4$, $S D=3.5$; Wilcoxon: $\mathrm{Z}=-6.1 ; p<.001$ ) als auch in Gruppe 2 (Mütter: $M=0.6, S D=1.4$; Jugendliche: $M=3.2, S D=3.7$; Wilcoxon: $\mathrm{Z}=-6.8 ; p<.001)$ erheblich unterschätzen. Weiterhin bestand kein signifikanter Zusammenhang zwi-

Tabelle 3. Längsschnittanalyse Risiko- und Schutzfaktoren im Kindesalter (Prä): Ergebnisse der multivariaten binären logistischen Regression (noch nie getrunken/nicht regelmäßiger vs. regelmäßiger) Alkoholkonsum

\begin{tabular}{|c|c|c|c|c|c|c|c|c|}
\hline & $B$ & $S D$ & Wald & $p$ & OR & $d$ & $95 \%-\mathrm{KI} O \mathrm{R}$ & VIF \\
\hline Alter des Kindes & 1.21 & 0.2 & 48.3 & $.000 * \star *$ & 3.35 & 0.67 & $2.4-4.7$ & 1.08 \\
\hline $\begin{array}{l}\text { Triple P-Teilnahme der Eltern } \\
(0=\text { keine Teilnahme, } 1=\text { Teilnahme })\end{array}$ & 0.46 & 0.3 & 2.0 & .153 & 1.58 & 0.25 & $0.8-3.0$ & 1.07 \\
\hline $\begin{array}{l}\text { Geschlecht des Kindes } \\
(0=\text { Mädchen, } 1=\text { Jungen })\end{array}$ & 0.34 & 0.3 & 1.2 & .283 & 1.40 & 0.19 & $0.8-2.6$ & 1.04 \\
\hline $\begin{array}{l}\text { Elterliche Probleme mit Alkohol } \\
(0=\text { nein, } 1=\mathrm{ja})\end{array}$ & -0.43 & 0.6 & 0.6 & .431 & 0.65 & -0.24 & $0.2-1.9$ & 1.07 \\
\hline $\begin{array}{l}\text { Mütterliches dysfunktionales Erziehungs- } \\
\text { verhalten }\end{array}$ & 0.21 & 0.3 & 0.4 & .510 & 1.23 & 0.11 & $0.7-2.3$ & 1.24 \\
\hline Psychische Beschwerden der Mutter & 0.01 & 0.0 & 0.6 & .424 & 1.01 & 0.01 & $1.0-1.0$ & 1.32 \\
\hline Internalisierende Auffälligkeiten des Kindes & -0.06 & 0.0 & 6.1 & $.014^{\star}$ & 0.94 & -0.03 & $0.9-1.0$ & 2.23 \\
\hline Externalisierende Auffälligkeiten des Kindes & 0.03 & 0.0 & 1.1 & .298 & 1.03 & 0.02 & $1.0-1.1$ & 2.72 \\
\hline Kindliches Temperament (Unruhe/Ärger) & -0.04 & 0.1 & 0.6 & .423 & 0.96 & -0.02 & $0.9-1.1$ & 1.47 \\
\hline $\begin{array}{l}\text { Kindliches Temperament (Annäherung/ } \\
\text { Exploration) }\end{array}$ & -0.01 & 0.1 & 0.0 & .849 & 0.99 & -0.01 & $0.9-1.1$ & 1.26 \\
\hline
\end{tabular}

Anmerkungen. ${ }^{\star} p<.05,{ }^{*} p<.01, * * * p<.001 ;$ VIF: Varianz-Inflations-Faktoren. 
schen der Mutter- und Jugendlicheneinschätzung (Gruppe $1: r=.06, p=.489$; Gruppe 2: $r=.05, p=.586$ ).

\section{Diskussion}

Im Rahmen des Zukunft Familie Projekts wurden Daten von Kindern vom Kindergarten- bis zum Jugendalter erhoben, was Längsschnittanalysen über einen Zeitraum von zehn Jahren ermöglicht. Dies ist insbesondere bedeutsam für die Vorhersage des jugendlichen Konsumverhaltens auf der Grundlage frühkindlicher Faktoren. Anhand der Ergebnisse der Studie sollen nun Ansatzpunkte für präventive Maßnahmen diskutiert werden. In der folgenden Darstellung sollte beachten werden, dass die Repräsentativität der Stichprobe aufgrund der geringen Stichprobengröße und der Stichprobenart (nur Kindergartenkinder im Rahmen einer Interventionsstudie; mittlere und obere Sozialschicht überrepräsentiert) eingeschränkt ist.

Bei der deskriptiven Auswertung der Daten zeigte sich, dass $46 \%$ der Jugendlichen in dieser Stichprobe bereits einen regelmäßigen Konsum von Alkohol aufwiesen, dabei zeigten sich keine Unterschiede in den Konsummustern zwischen Mädchen und Jungen. Das Erstkonsumalter von 13.5 Jahren lag bei den regelmäßig Trinkenden in dieser Studie deutlich unter dem gesetzlichen Mindestalter. Weiterhin berichteten von den regelmäßig Konsumierenden in dieser Stichprobe rund 31\% von Rauscherfahrungen in den letzten drei Monaten und jeder vierte hatte Binge Drinking in den letzten 30 Tagen betrieben.

Auch wenn in dieser Studie der Zusammenhang zwischen elterlichen Regeln und dem jugendlichen Alkoholkonsum nicht untersucht wurde, vermuten wir, da rund $29 \%$ der befragten Elternteile angaben, keine Regeln in Bezug auf den jugendlichen Alkoholkonsum zu haben, dass das Aufstellen von Regeln vor einem frühzeitigen problematischen Konsum im Jugendalter schützen könnte (bspw. Koning, van den Eijnden, Verdurmen, Engels \& Vollebergh, 2012). Dies könnte eine Grundlage für zukünftige Studien darstellen.

In Hinblick auf die Soziodemographie (Fragestellung 1) zeigte sich, dass Kinder, welche in einem Haushalt aus einer höheren sozialen Schicht aufwachsen, ein erhöhtes Risiko für den regelmäßigen Konsum im Jugendalter aufweisen. $\mathrm{Zu}$ diesem Ergebnis kommt auch die englische Avon-Längsschnittstudie (Kendler et al., 2014). Das biologische kindliche Geschlecht, der Erziehungsstatus sowie ein familiärer Migrationshintergrund zeigten, im Gegensatz zu unserer Vermutung, keine signifikanten Zusammenhänge zum Alkoholkonsum im Jugendalter auf.

Mit Ausnahme der internalen kindlichen Auffälligkeiten leisteten die untersuchten frühkindlichen Risikofaktoren
(Fragestellung 2) aus Sicht der Mütter, entgegen unserer Vermutung, keinen bedeutsamen Beitrag zur Vorhersage des Alkoholkonsums in dieser Stichprobe. Es wurden Hinweise dafür gefunden, dass internale Auffälligkeiten im Kindesalter vor einem regelmäßigen Konsum im Jugendalter schützen. $\mathrm{Zu}$ diesem Ergebnis kommen auch Heradstveit et al. (2018). In ihrer Längsschnittstudie schützen internalisierende Störungen im Kindesalter vor einem jugendlichen Konsum, während externale kindliche Auffälligkeiten den Konsum im Jugendalter förderten. Letzteres konnte in dieser Studie hingegen nicht bestätigt werden, was im Widerspruch zur bisherigen Literatur steht (z.B. Miettunen et al., 2014). Eine mögliche Begründung von Edwards et al. (2014) dafür, dass internale Auffälligkeiten einen Schutzfaktor darstellen ist, dass in der frühen Kindheit derartige Auffälligkeiten mit einem verhaltenshemmenden Temperament assoziiert sein könnten, was den Aufbau von engen sozialen Kontakten zu Peers erschwert. Da in der Jugend Peerbeziehungen bezüglich des Alkoholkonsums eine große Rolle spielen, ist die Wahrscheinlichkeit dann geringer, dass Kinder sich unter Gleichaltrigen wiederfinden, die mit Alkohol experimentieren.

Dass kindliche Faktoren sich nicht als signifikante Prädiktoren erwiesen, resultiert vermutlich zum einen aus dem geringen Alter der untersuchten Jugendlichen $(M=$ 14) und ihrem, im Vergleich zu Älteren, vergleichsweise geringen Konsum. Zum anderen wurden nur Prädiktoren aus dem 10 Jahre zurückliegenden Kindergartenalter und aus der Sicht der Mütter einbezogen. Die Prädiktoren aus der väterlichen Sicht und die Entwicklung der Faktoren über die Zeitspanne hinweg wurden nicht untersucht. Insbesondere Letzteres könnte jedoch bedeutsam sein, da Blomeyer, Laucht, Hohm, Dyer und Schmidt (2007) in ihrer Studie zeigen konnten, dass bspw. Störungen des Sozialverhaltens erst ab einem Erstmanifestationsalter von acht Jahren in Verbindung mit einem exzessiveren Alkoholkonsum im Jugendalter stehen.

Hinsichtlich der Trinkmotive (Fragestellung 3) zeigte sich innerhalb unserer Stichprobe, dass sowohl die sozialen Motive als auch die Verstärkungsmotive am stärksten bei den Jugendlichen ausgeprägt waren, gefolgt von Bewältigungs- und Konformitätsmotiven. Dies stimmt mit unserer Annahme und der Literatur überein (Kuntsche \& Kuntsche, 2009). Sowohl soziale Motive als auch Verstärkungs- und Bewältigungsmotive korrelierten signifikant mit der Konsummenge aus Jugendlichensicht, Konformitätsmotive hingegen nicht. Eine vermehrte Ausprägung der Motive war dabei mit einem vermehrten Alkoholkonsum assoziiert. Bei Wurdak, Dörfler, Eberhard und Wolstein (2010) lagen die Verstärker- und sozialen Motive ebenfalls vorn, wobei in ihrer Studie alle Dimensionen signifikant positiv mit der Konsummenge korrelierten. Jugendliche trinken dementsprechend insbesondere um in- 
ternale („Spaß haben“) und externale (,Zusammenkünfte mehr genießen“) positive Verstärkungen zu erfahren. Präventionsmaßnahmen könnten die Jugendlichen für die Trinkmotive sensibilisieren und Alternativen aufzeigen, um diese positiven Verstärkungen auf risikofreien Wegen zu erreichen (bspw. feiern und tanzen in der Disco ohne Alkoholkonsum).

In der vorliegenden Studie unterschätzten Mütter den Konsum ihrer Kinder erheblich (Fragestellung 4). Die mütterlichen und kindlichen Einschätzungen wiesen keinen signifikanten Zusammenhang auf. Dies steht in Kongruenz zur Literatur (bspw. Lampert \& Thamm, 2007). In entsprechenden präventiven Maßnahmen könnten Mütter geschult werden, Hinweise frühzeitig zu erkennen, die möglicherweise auf einen übermäßigen Alkoholkonsum ihrer Kinder hindeuten. Guilamo-Ramos, Jaccard, Turrisi, Johansson und Bouris (2006) kommen ebenfalls zu dem Ergebnis, dass Mütter den Alkoholkonsum teils stark unterschätzen. Sie schlagen vor, dass Präventionsprogramme Eltern dafür sensibilisieren sollten, dass ein Jugendlicher nicht dem Trinker-Stereotyp entsprechen muss, um trotzdem regelmäßiger Konsument zu sein (z. B. „Mein Kind ist gut in der Schule und kann deshalb gar nicht trinken."). Weiterhin könnte das sogenannte Parental Monitoring (= Eltern wissen was ihr Kind mit wem in der Freizeit unternimmt) vor einem Beginn des problematischen Alkoholkonsums schützen (bspw. Rusby, Light, Crowley \& Westling, 2018). Durch das verbesserte Wissen über die kindliche Freizeitgestaltung könnten die mütterlichen Einschätzungen gleichzeitig auch realistischer ausfallen.

Zusammenfassend lässt sich festhalten, dass Präventionsmaßnahmen den Jugendlichen Alternativen aufzeigen können, um die sozialen und verstärkenden Motivationen des Trinkens auf risikofreien Wegen zu erreichen. Zur verbesserten Einschätzung des kindlichen Konsums, können Mütter in Hinweisen geschult werden, die auf einen übermäßigen Konsum hindeuten. Das erhöhte Risiko von Kindern aus Familien mit einem höheren sozialen Status könnte dabei entsprechend berücksichtigt werden.

\section{Limitationen und Fazit}

Die Ableitung allgemeiner präventiver Ideen anhand unserer Ergebnisse ist nicht nur durch die Stichprobenart und die geringe Stichprobengröße (s.o.) eingeschränkt, sondern auch dadurch, dass bei der Einschätzung des kindlichen Problemverhaltens nur die Perspektive der Mutter in den Analysen berücksichtigt wurde. Wünschenswert wäre es, in künftigen Studien zusätzlich Väterangaben einzubeziehen. Weiterhin muss damit gerechnet werden, dass die Erhebung des jugendlichen Alkoholkonsums durch Selbstauskünfte bspw. durch soziale Erwünschtheit zum Teil verzerrt ist, auch wenn die Jugendlichen nachdrücklich darauf hingewiesen wurden, dass ihre Eltern ihre Angaben nicht erfahren. Dies sind wahrscheinlich auch die Gründe dafür, dass nur einige wenige signifikante Effekte in Hinblick auf die soziodemografischen und frühkindlichen Faktoren aufgedeckt werden konnten.

Die Stärke unserer Studie besteht darin, dass es sich um eine 10-Jahres-Längsschnittstudie handelt. Hierbei wurde jedoch nur der Prä-Messzeitpunkt für die Ermittlung von frühkindlichen Prädiktoren beachtet, während die Entwicklung der Faktoren über die Zeitspanne hinweg nicht analysiert wurde.

In zukünftigen Studien wäre es wünschenswert anhand einer repräsentativen Stichprobe die Entwicklung der einzelnen Faktoren über die frühe Kindheit hinweg getrennt für Jungen und Mädchen sowie für unterschiedliche $\mathrm{Al}$ tersbereiche zu betrachten.

Nach Arnaud und Thomasius (2020) wird das familiäre Setting noch nicht ausreichend zur Umsetzung von (sucht-)präventiven Maßnahmen in Deutschland genutzt. Die Ergebnisse dieser Studie liefern Hinweise dafür, dass die Trinkmotive der Jugendlichen sowie die abweichende mütterliche Einschätzung des jugendlichen Konsums mögliche Ansatzpunkte für präventive Maßnahmen darstellen könnten. Die bisherige Studienlage zu diesen Aspekten, insbesondere im deutschen Raum, fällt gering aus, sodass weitere Forschung zu diesen Aspekten notwendig und wünschenswert ist.

\section{Literatur}

Arnaud, N. \& Thomasius, R. (2020). Prävention von stoffgebundenen Suchtstörungen. Zeitschrift für Kinder- und Jugendpsychiatrie und Psychotherapie, 48 (5), 381-392. https://doi.org/10. 1024/1422-4917/a000636

Blomeyer, D., Laucht, M., Hohm, E., Dyer, A.S. \& Schmidt, M.H. (2007). Exzessiver Alkoholkonsum bei 15-Jährigen mit externalen Störungen im Kindes- und Jugendalter. Zeitschrift für Psychiatrie, Psychologie und Psychotherapie, 55 (3), 145-154. https://doi.org/10.1024/1661-4747.55.3.145

Brownlie, E., Beitchman, J.H., Chaim, G., Wolfe, D.A., Rush, B. \& Henderson, J. (2019). Early adolescent substance use and mental health problems and service utilization in a school-based sample. The Canadian Journal of Psychiatry, 64 (2), 116-125. https://doi.org/10.1177/0706743718784935

Dawson, D.A., Goldstein, R. B., Chou, S.P., Ruan, W.J. \& Grant, B.F. (2008). Age at first drink and the first incidence of adult-onset DSM-IV alcohol use disorders. Alcoholism: Clinical and Experimental Research, 32 (12), 2149-2160. https://doi.org/10.1111/j. 1530-0277.2008.00806.x

Döpfner, M., Plück, J., Kinnen, C. \& Arbeitsgruppe Deutsche Child Behavior Checklist. (2014). Manual deutsche Schulalter-Formen der Child Behavior Checklist von Thomas M. Achenbach. Elternfragebogen über das Verhalten von Kindern und Jugendlichen, (CBCL/6-18R), Lehrerfragebogen über das Verhalten von Kindern und Jugendlichen (RF/6-18R), Fragebogen für Jugendliche (YSR/11-18R). Göttingen: Hogrefe. 
Edwards, A.C., Latendresse, S.J., Heron, J., Bin Cho, S., Hickman, M., Lewis, G. et al. (2014). Childhood internalizing symptoms are negatively associated with early adolescent alcohol use. Alcoholism Clinical and Experimental Research, 38, 16801688. https://doi.org/10.1111/acer.12402

Guilamo-Ramos, V., Jaccard, J., Turrisi, R., Johansson, M. \& Bouris, A. (2006). Maternal perceptions of alcohol use by adolescents who drink alcohol. Journal of Studies on Alcohol and Drugs, 67 (5), 730-737. https://doi.org/10.15288/jsa.2006.67.730

Hahlweg, K. \& Schulz, W. (2018). Universelle Prävention kindlicher Verhaltensstörungen durch Elterntrainings. Wirksamkeit nach 10 Jahren aus Sicht der Mütter, Väter und Jugendlichen. Zeitschrift für Klinische Psychologie und Psychotherapie, 47 (1), 1-15. https://doi.org/10.1026/1616-3443/a000462

Heradstveit, O., Skogen, J.C., Bøe, T., Hetland, J., Pedersen, M.U. \& Hysing, M. (2018). Prospective associations between childhood externalising and internalising problems and adolescent alcohol and drug use: The Bergen Child Study. Nordic Studies on Alcohol and Drugs, 35 (5), 357-371. https://doi.org/10.1177/14550 72518789852

Kendler, K.S., Gardner, C. O., Hickman, M., Heron, J., Macleod, J., Lewis, G. et al. (2014). Socioeconomic status and alcohol-related behaviors in the Avon longitudinal study of parents and children. Journal of Studies on Alcohol and Drugs, 75 (4), 541545. https://doi.org/10.15288/jsad.2014.75.541

Kuntsche, E. \& Kuntsche, S. (2009). Development and validation of the Drinking Motive Questionnaire Revised Short Form (DMQ-R SF). Journal of Clinical Child \& Adolescent Psychology, 38 (6), 899-908. https://doi.org/10.1080/15374410903258967

Koning, I.M., van den Eijnden, R.J.J.M., Verdurmen, J.E.E., Engels, R.C.M.E. \& Vollebergh, W.A.M. (2012). Developmental alcoholspecific parenting profiles in adolescence and their relationships with adolescents' alcohol use. Journal of Youth and Adolescence, 41 (11), 1502-1511. https://doi.org/10.1007/s10964-012-9772-9

Köppe, E. (2001). Glückliche Eltern, liebe Kinder: Auswirkungen von Partnerschaft und psychischer Symptomatik der Eltern auf das Verhalten ihrer Kinder. Unveröffentlichte Dissertation, Technische Universität Braunschweig.

Lampert, T. \& Thamm, M. (2007). Tabak-, Alkohol- und Drogenkonsum von Jugendlichen in Deutschland. Bundesgesundheitsblatt - Gesundheitsforschung - Gesundheitsschutz, 50, 600-608. https://doi.org/10.1007/s00103-007-0221-y

Lichtsinn, N. (2013). Kindliches Temperament: Struktur und Zusammenhänge zu Verhaltensauffälligkeiten unter Berücksichtigung mütterlicher Merkmale. Unveröffentlichte Dissertation, Technische Universität Braunschweig.

Maggs, J.L., Patrick, M.E. \& Feinstein, L. (2008). Childhood and adolescent predictors of alcohol use and problems in adolescence and adulthood in the National Child Development Study. Addiction, 103 (Suppl. 1), 7-22. https://doi.org/10.1111/j.13 60-0443.2008.02173.x

Meruelo, A.D., Castro, N., Cota, C.I. \& Tapert, S. F. (2017). Cannabis and alcohol use, and the developing brain. Behavioural Brain Research, 325 (Part A), 44-50. https://doi.org/10.1016/j.bbr.20 17.02.025

Miettunen, J., Murray, G. K., Jones, P. B., Mäki, P., Ebeling, H., Taanila, A. et al. (2014). Longitudinal associations between childhood and adulthood externalizing and internalizing psychopathology and adolescent substance use. Psychological Medicine, 44 (8), 1727-1738. https://doi.org/10.1017/S0033291713002328

Naumann, S., Bertram, H., Kuschel, A., Heinrichs, N., Hahlweg, K. \& Döpfner, M. (2010). Der Erziehungsfragebogen (EFB) - Ein Fra- gebogen zur Erfassung elterlicher Verhaltenstendenzen in schwierigen Erziehungssituationen. Diagnostica, 56 (3), 144157. https://doi.org/10.1026/0012-1924/a000018

Orth, B. \& Merkel, C. (2020). Die Drogenaffinität Jugendlicher in der Bundesrepublik Deutschland 2019. Rauchen, Alkoholkonsum und Konsum illegaler Drogen: Aktuelle Verbreitung und Trends. BZgA-Forschungsbericht. Köln: Bundeszentrale für gesundheitliche Aufklärung.

Reinherz, H.Z., Giaconia, R.M., Hauf, A.M.C., Wasserman, M.S. \& Paradis, A. D. (2000). General and specific childhood risk factors for depression and drug disorders by early adulthood. Journal of the American Academy of Child \& Adolescent Psychiatry, 39 (2), 223-231. https://doi.org/10.1097/00004583-200002000-0 0023

Rusby, J.C., Light, J. M., Crowley, R. \& Westling, E. (2018). Influence of parent-youth relationship, parental monitoring, and parent substance use on adolescent substance use onset. Journal of Family Psychology, 32 (3), 310-320. https://doi.org/10.1037/ fam0000350

Sanders, M. R. (2012). Development, evaluation, and multinational dissemination of the Triple P-Positive Parenting Program. Annual Review of Clinical Psychology, 8, 345-379. https://doi. org/10.1146/annurev-clinpsy-032511-143104

Wurdak, M., Dörfler, T., Eberhard, M. \& Wolstein, J. (2010). Tagebuchstudie zu Trinkmotiven, Affektivität und Alkoholkonsum bei Jugendlichen. SUCHT, 56, 175-182. https://doi.org/10.1024/ 0939-5911/a000027

\section{Finanzielle Unterstützung}

Die Projekte Zukunft Familie I und III wurden von der Deutschen Forschungsgemeinschaft finanziell gefördert (ZF I; HA 1400/141-3; 4-5; ZF III; HA 1400/17-1).

\section{Förderung}

Open-Access-Veröffentlichung ermöglicht durch TU Braunschweig.

\section{Deklaration konkurrierender Interessen}

Es bestehen für Max Supke und Wolfgang Schulz keine Interessenkonflikte.

\section{Einhaltung ethischer Richtlinien}

Die Ethikkommission der Deutschen Gesellschaft für Psychologie hat das Forschungsvorhaben mit Votum vom 20.01.2011 als „ethisch unbedenklich“ bewertet (Kennnummer:WS 12_2010). Die Teilnahme an der Studie war freiwillig. Die Eltern und Jugendlichen wurden über die Ziele und Inhalte der Studie sowie über den Datenschutz informiert und gaben ihr schriftliches Einverständnis.

\section{Sc. Max Supke}

Institut für Psychologie

Technische Universität Braunschweig

Humboldtstraße 33

38106 Braunschweig

Deutschland

m.supke@tu-bs.de 